\title{
Análise de transferência de calor em um reator trifásico de leito fixo com aplicação da transfor- mada de Laplace
}

\author{
Analysis of the heat transfer in a tickle bed reactor with application of Laplace \\ Transform
}

\author{
Evanderson Heleno do Aguiar \\ Escola Politécnica de Pernambuco \\ Universidade de Pernambuco \\ 50.720-001 - Recife, Brasil \\ evandersonaguiar@hotmail.com
}

\author{
Antonio Carlos L. Lins Cavalcanti \\ Escola Politécnica de Pernambuco \\ Universidade de Pernambuco \\ 50.720-001 - Recife, Brasil \\ antoniocarlosllinscs@hotmail.com
}

\author{
Jornandes Dias da Silva \\ Escola Politécnica de Pernambuco \\ Universidade de Pernambuco \\ 50.720-001 - Recife, Brasil \\ jornandesdias@poli.br
}

Resumo previsões quantitativas do comportamento transiente de processos catalíticos fluido-sólido têm sido
feitas a partir de modelos fenomenológicos, tendo em vista a adequação de seus proje-tos, a extrapola-
ção em escala, operações e controle. Neste trabalho, apresenta-se a modelagem fenomenológica para o
reator trifásico sob regime de leito gotejante, abordando as restriçôes para este regime, aplicaçães de
modelos e os respectivos parâmetros hidrodinâmicos. O desen-volvimento operacional da pesquisa de
implantação de um processo reativo catalítico trifásico é descrito obedecendo à ordem de etapas que
seguem o processo. Como ferramenta matemática utilizou-se a Transformada de Laplace (TL) para a
transformação das equaçôes diferenciais parciais do modelo em equaçães diferenciais ordinárias. Logo
em seguida foi aplicada técnica numérica da inversão da TL, onde se utilizou linguagens de programa-
ção como Fortran e Ma-tlab para modelagem de perfis que descrevem o comportamento do reator em
função do tempo e da distância radial. Como resultado do projeto, obtiveram-se resultados numéricos
que mode-lam o comportamento termodinâmico em uma seção do reator, através das variáveis tempo e
distância radial, nas fases sólida e gasosa, relacionando-as através de perfis tridimensionais

Palavras-Chave: Reator, leito fixo, Transformada de Laplace

\begin{abstract}
The quantitative predictions about the transient behavior of fluid-solid catalytic processes have been made from phenomenological models, according with the adequation of their projects, to the extrapolation on scale, operations and controls. In this paper is presented the phenomenological modeling for a trickle bed reactor operating in trickle flow pattern, considering the restrictions for this pattern, applications of models and their hydrodynamic parameters. The operational development of the research about the implantation of a three-phases catalytic reactive process is showed following the order of steps of the process. As a mathematical tool, was used the Laplace Transform (LP) to transform the Partial Differential Equations (PDE), that describes the model, in Ordinary Differential Equations $(O D E)$. After that, was utilized a numerical technique to invert the LP, when was used programming languages like Fortran and Matlab, for modeling of profiles that describes the behavior of the reactor over the time and the axial distance. As result, it was obtained numerical results that models the thermodynamic behavior in a section of the reactor through the variables time and distance, in the gas and solid phases, relating them through three-dimensional profiles.
\end{abstract}

Keywords: Reactor, trickle bed, Laplace Transform 


\section{Introdução}

Os reatores trifásicos de leito fixo têm uma ampla aplicação no setor industrial, sendo usado em indústrias de petróleo e processos de refinaria, tal como hidrodessulfurização e hidrogenação mas também pode ser aplicado na oxidação de matéria orgânica em águas residuais e reações enzimáticas [5]. As variações das vazões de gás e líquido podem causar mudanças nos regimes de escoamento durante as operações em reatores trifásico de leito fixo. Estes reatores operam numa faixa de velocidade superficial de líquido entre $10-4 \mathrm{~m} / \mathrm{s}$ a $3 \times 10-3 \mathrm{~m} / \mathrm{s}$ em reatores de plantas - piloto e entre $10-3 \mathrm{~m} / \mathrm{s}$ a $2 \times 10-2 \mathrm{~m} / \mathrm{s} \mathrm{em}$ reatores comerciais [2]. Quanto à velocidade superfi-cial do gás, situa-se entre os valores de $2 \times 10-2 \mathrm{~m} / \mathrm{s}$ a $4,5 \times 10-1$ $\mathrm{m} / \mathrm{s}$ para plantas - piloto e $1,5 \times 10-1 \mathrm{~m} / \mathrm{s}$ a $3 \mathrm{~m} / \mathrm{s}$ para reatores comerciais.

Essa variação de comportamento são classificadas em três tipo de regime: Regime de Leito Gotejante, Pulsante e de Bolhas dispersas. O Regime de Leito Gotejante é o mais utilizado em aplicações industriais devido a sua alta conversão e fácil adaptação para altas pressões, maior fator de efetividade, entre outras vantagens [3].

O conhecimento do comportamento termodinâmico ao longo do reator é de fundamental importância no momento em que este é projetado, ou até mesmo na fase de controle do reator já em funcionamento. Neste trabalho se estudou o comportamento de um reator de leito fixo operando em regime de leito gotejante.

\section{Metodologia}

O fenômeno de transferência de calor que ocorre dentro do reator poder ser entendido através das seguintes relações

Para fase sólida:

$$
\frac{\partial T_{s}(r, t)}{\partial t}=\frac{k_{s}}{C_{p, s} \rho_{s}}\left(\frac{\partial^{2} T_{s}(r, t)}{\partial r^{2}}+\frac{2}{r} \frac{\partial T_{s}(r, t)}{\partial r}\right)
$$

Com as seguintes condições de contorno:

$$
\begin{aligned}
& \left.T_{s}(r, t)\right|_{t=0}=0 ; T_{s} \in(0, R) e T_{s} \in\left(t_{0}, t_{\text {final }}\right) \\
& \left.k_{s} \frac{\partial T_{s}(r, t)}{\partial r}\right|_{R=r}=h_{s}\left(T_{g}(z, t)-\left.T_{s}(r, t)\right|_{R=r}\right) ; t \geq 0 \\
& \left.\frac{\partial T_{s}(r, t)}{\partial r}\right|_{r=0}=0 ; t \geq 0
\end{aligned}
$$

Para a fase gasosa:

$$
\begin{aligned}
& \frac{\partial T_{g}(z, t)}{\partial t}+V_{s g} \frac{\partial T_{g}(z, t)}{\partial z}=\alpha_{a x, g} \frac{\partial^{2} T_{g}(z, t)}{\partial z^{2}}- \\
& \left.\frac{3 k_{s}}{R C_{p, g} \rho_{g}} \frac{\left(1-\varepsilon_{b}\right)}{\varepsilon_{b}}\left(\frac{\partial T_{s}(r, t)}{\partial r}\right)\right|_{R=r}
\end{aligned}
$$

Com as seguintes condições de contorno

$$
\begin{aligned}
& \left.T_{g}(z, t)\right|_{t=0}=0 ; T_{g} \in(0, L) e T_{g} \in\left(t_{0}, t_{\text {final }}\right) \\
& \left.T_{g}(z, t)\right|_{z=0^{+}}=T_{g, 0} ; t \geq 0 \\
& \left.T_{g}(z, t)\right|_{z=\infty}=0 ; t \geq 0
\end{aligned}
$$

As equações (1) e (5) descrevem o fenômeno da transferência de calor dentro do reator. Estas são equações diferenciais parciais que devido ao seu grau de complexidade não podem ser resolvidas analiticamente. Como solução foi adotada a técnica da transformada de Laplace e sua inversa, para resolução das equações. Aplicando a Transformada de Laplace às equações (1) e (5) em rela-ção à variável, obtém-se as equações (9) e (10), respec-tivamente, que são equações diferenciais ordinárias de segunda, resolvíveis analiticamente.

$$
\begin{aligned}
& \frac{d^{2} T_{s}(r, s)}{d r^{2}}+\frac{2}{r} \frac{d T_{s}(r, s)}{d r}-\frac{C_{p, s} \rho_{s}}{k_{s}} s T_{s}(r, s)=0 \\
& s T_{g}(z, s)-T_{g}(z, 0)+V_{s g} \frac{d T_{g}(z, s)}{\partial d z}= \\
& \alpha_{a x, g} \frac{d^{2} T_{g}(z, s)}{d z^{2}}-\frac{3 k_{s}}{R C_{p, g} \rho_{g}} \frac{\left(1-\varepsilon_{b}\right)}{\varepsilon_{b}}\left(\frac{d T_{s}(r, s)}{d r}\right)_{R-r}
\end{aligned}
$$

As soluções para as equações (9) e (10), são dadas nas equações (11) e (12), respectivamente.

$$
T_{s}(r, s)=\frac{1}{r} \beta(s) \sinh \left(r \sqrt{\frac{C_{p, s} \rho_{s}}{k_{s}} s}\right) T_{g}(z, s)
$$


Onde

$$
\begin{aligned}
& \beta(s)=\frac{R h_{s}}{\left[\begin{array}{l}
k_{s} \sqrt{\frac{C_{p, s} \rho_{s}}{k_{s}} s \cosh \left(R \sqrt{\frac{C_{p, s} \rho_{s}}{k_{s}} s}\right)+} \\
\sinh \left(R \sqrt{\frac{C_{p, s} \rho_{s}}{k_{s}} s}\right)\left(h_{s}-\frac{k_{s}}{R}\right)
\end{array}\right]} \\
& T_{g}(z, s)=e^{z\left(\frac{V_{g g}}{2 \alpha_{a r, g}}\right)}\left[\begin{array}{l}
\frac{T_{g, 0}}{s} \cosh (z \delta)- \\
\frac{T_{g, 0}}{s} \operatorname{coth}(\delta) \sinh (z \delta)
\end{array}\right]
\end{aligned}
$$

Onde

$$
\begin{aligned}
& \delta(s)=\frac{\sqrt{V_{s g}^{2}+4 \alpha_{a x, g}(\xi(s)+s)}}{2 \alpha_{a x, g}} \\
& \xi(s)=\frac{3 k_{s}}{R C_{p, g} \rho_{g}} \frac{\left(1-\varepsilon_{b}\right)}{\varepsilon_{b}} \phi(s) \\
& \phi(s)=\left[\begin{array}{l}
R \beta(s) \sqrt{\frac{C_{p, s} \rho_{s}}{k_{s}} s \cosh \left(R \sqrt{\frac{C_{p, s} \rho_{s}}{k_{s}} s}\right)}- \\
\beta(s) \sinh \left(R \sqrt{\frac{C_{p, s} \rho_{s}}{k_{s}} s}\right) \\
R^{2}
\end{array}\right] \\
& \beta(s)=\frac{R h_{s}}{\left[\begin{array}{l}
k_{s} \sqrt{\frac{C_{p, s} \rho_{s}}{k_{s}}} s \cosh \left(R \sqrt{\frac{C_{p, s} \rho_{s}}{k_{s}} s}\right)+ \\
\sinh \left(R \sqrt{\frac{C_{p, s} \rho_{s}}{k_{s}} s}\right)\left(h_{s}-\frac{k_{s}}{R}\right)
\end{array}\right]}
\end{aligned}
$$

Obteve-se portanto, funções e em função da variável de Laplace. O procedimento a ser adotado neste momento é a aplicação da transformada inversa de Laplace para que as funções voltem à variável inicial, . $\mathrm{s}$ T g T s t
A Transformada Inversa de Laplace pode ser obtida através da equação (13) para fase sólida e da equação (14) para a fase gasosa.

$$
\begin{aligned}
& T_{s}(r, t)=\frac{1}{2 \pi i} \lim _{T \rightarrow \infty} \int_{\gamma-i T}^{\gamma+i T} T_{s}(r, s) e^{s t} d s \\
& T_{g}(z, t)=\frac{1}{2 \pi i} \lim _{T \rightarrow \infty} \int_{\gamma-i T}^{\gamma+i T} T_{g}(z, s) e^{s t} d s
\end{aligned}
$$

Essa é uma integral complexa, Conhecida por Fórmula inversa de Mellin, Integral de Bromwitch ou até mesmo Integral de Fourier-Mellin. A variável $s$ de Laplace é uma variável complexa, ou seja, $s=\gamma+i \omega$. A integal acima pode ser entendida como uma integral de linha sobre um caminho que liga os pontos $(\gamma-i T)$ e $(\gamma+i T)$; pelo teorema de Cauchy, o qual diz que a integral sobre um caminho que liga dois pontos independe do caminho escolhido, pode-se escolher o caminho mais simples para se trabalhar, então pode-se considerar a reta vertical $x=\gamma$ para ligar os pontos acima mencionados. Sendo assim, as equações paramétricas de ${ }^{s}$ são:

$$
s(\omega)=\left\{\begin{array}{l}
x=\gamma \\
y=i \omega
\end{array}, \omega \in[-T, T]\right.
$$

Pela definição de integral de linha:

$$
\int_{c} f(z) d z=\int_{a}^{b} f(z(t)) z^{\prime}(t) d t
$$

Onde $c$ é um caminho definido por uma função $z(t)$, $\mathrm{e}$ os limites de integração $(a, b)$ forma o intervalo de variação da variável $t$. Aplicando essa ideia às equações (13) e (14), lembrando que a temperatura é uma variável real, obtém-se as equações (15) e (16).

$$
\begin{aligned}
& T_{s}(r, t)=\frac{e^{t \gamma}}{\pi} \int_{0}^{\infty} \operatorname{Re}\left[I_{s}(r, s(\omega)) e^{n \omega}\right] d \omega \\
& T_{g}(z, t)=\frac{e^{t \gamma}}{\pi} \int_{0}^{\infty} \operatorname{Re}\left[I_{g}(z, s(\omega)) e^{\pi \omega \omega}\right] d \omega
\end{aligned}
$$

Para resolver as equações (15) e (16), foi desenvolvido um código computacional utilizando a linguagem de programação Fortran, o qual utilizou o método numérico da Regra do Trapézio, através do qual foram obtidas as equaçōes iterativas (17) e (18) para as fases sólida e gasosa, respectivamente. 


$$
T_{s}(r, t)=\frac{h e^{t y}}{\pi}\left[\begin{array}{l}
\frac{\left[T_{r}(r, s(0))+\phi_{s}\right]}{2}+ \\
\sum_{k=1}^{n} \operatorname{Re}\left[T_{r}(r, s(k h)) e^{\pi i k h}\right]
\end{array}\right]
$$

$$
\begin{aligned}
& \text { Onde } \\
& \phi_{s}=\lim _{\omega \rightarrow j} \operatorname{Re}\left[T_{s}(r, s(\omega)) e^{t i \omega}\right] \\
& T_{g}(z, t)=\frac{h e^{t \gamma}}{\pi}\left[\begin{array}{l}
\frac{\left[T_{g}(z, s(0))+\phi_{g}\right]}{2}+ \\
\sum_{k=1}^{n} \operatorname{Re}\left[T_{g}(z, s(k h)) e^{t i k h}\right]
\end{array}\right]
\end{aligned}
$$

Onde

$$
\phi_{g}=\lim _{\omega \rightarrow j} \operatorname{Re}\left[T_{g}(z, s(\omega)) e^{\tilde{\omega} \omega}\right]
$$

\section{Resultados}

Como melhor forma de visualizar os resultados, o código Fortran produzido gerou arquivo de dados de saída para ser importado e plotado em gráficos tridimensionais através de um código desenvolvido através da linguagem Matlab. Os resultados apresentados nas figuras (1) e (2) representam o comportamento da temperatura ao longo do raio da partícula catalítica, na fase sólida, e ao longo do eixo do reator, na fase gasosa.

Percebe-se na Figura 1, que não há considerável variação de temperatura ao longo do raio da partícula catalisadora, enquanto que em relação ao tempo, percebe-se o crescimento da temperatura, com uma leve queda após um certo período de tempo. Quanto à Figura 2, nota-se que a temperatura em relação ao tempo varia de maneira semelhante à Figura 1, porém, ao longo do eixo do reator há notável decrescimento da temperatura, ou seja, na entrada do reator a temperatura tende a ser levemente superior que na saída do reator.

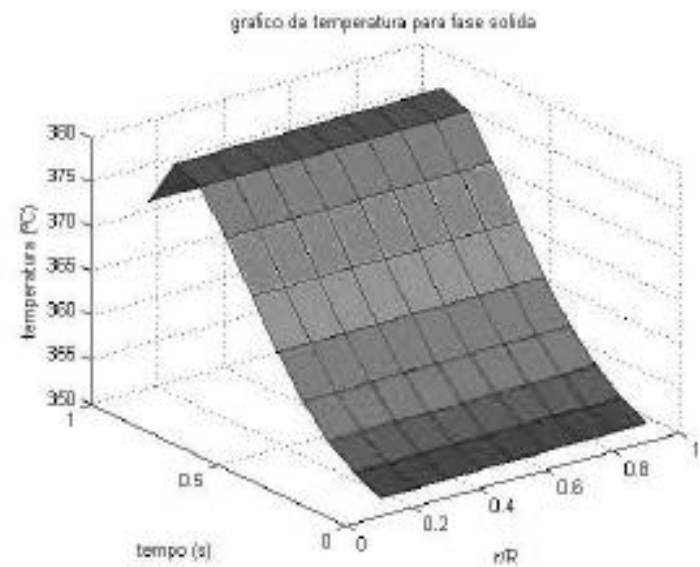

Figura 1 : Grafico mostrando o comportamento térmico ao longo do raio da particula catalisadorn (fase sólida)

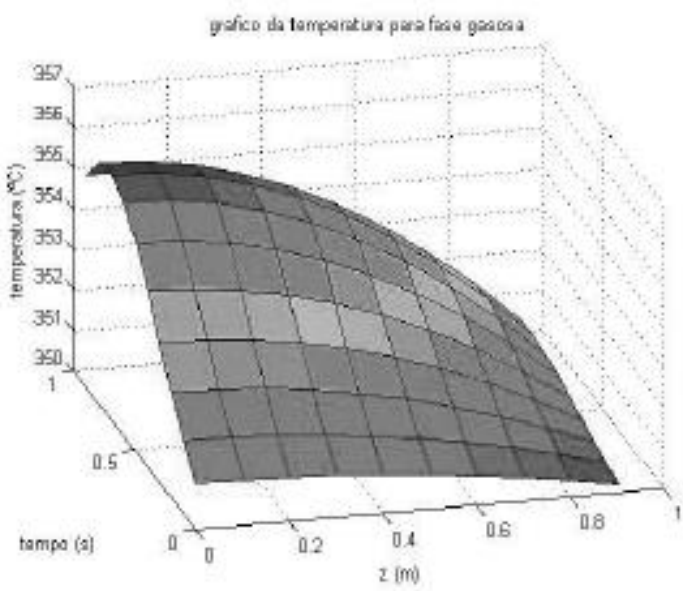

Figura 1 : : Grifico mostrando o comportamento téruico ao longo do eixo do reator (fase gasosa)

\section{Referências}

[1] Iliuta, I.; Bildea, S. C.; Iliuta, M. C.; Larachi, F. Analysis of Trickle Bed and Packed Bubble Column Bioreactors for Combined Carbon Oxidation and Nitrification. Brazilian Journal of Chemical Engineering. 19: 69-87, 2002.

[2] Ramachandran, P. A.; Chaudhari, R. B. ThreePhase Catalytic Reactors, Gordan and Breach, New York, U.S.A. 1983.

[3] Silva, J. D. Dynamic evaluation for liquid tracer in a trickle bed reactor. J. Braz. Soc. Mech. Sci. \& Eng., v. 33, n. 3, 2011. DOI: 10.1590/S1678-58782011000300002. 
[4] Silva, J.D.; Lima, F.R.A.; Abreu, C.A.M. and Knoechelmann, A. Experimental analysis and evaluation of the mass transfer process in a trickle-bed reactor. Braz. J. Chem. Eng., 2003, vol.20, n.4, pp. 375-390. DOI: 10.1590/S010466322003000400005 .

[5] Zeljko V. Milorad P. D. Computational Modeling of Trickle Bed Reactors. Industrial \& Engineering Chemistry Research. 2012. 51, 1663 1671. 